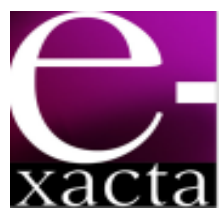

ISSN: 1984-3151

\title{
ABSORÇÃO INTRABANDA EM PONTOS QUÂNTICOS ESFÉRICOS: EFEITOS DE CAMPO MAGNÉTICO
}

\author{
INTRABAND ABSORPTION IN SPHERICAL QUANTUM DOTS: \\ MAGNETIC FIELD EFFECTS
}

\author{
Silvio José Prado \\ Faculdade de Ciências Integradas do Pontal - FACIP - UFU, Ituiutaba, MG. \\ sprado@pontal.ufu.br
}

Recebido em: 26/04/2011 - Aprovado em: 30/06/2011 - Disponibilizado em: 24/07/2011

\begin{abstract}
RESUMO: Neste trabalho foi feito um estudo dos diferentes aspectos das propriedades ópticas em um único ponto quântico esférico de CdTe na presença de um campo magnético externo B aplicado na direção-z, após ser realizado um estudo sistemático dos autovalores de energia, funções de onda e das simetrias dominantes dentro do modelo k.p $8 \times 8$ de Kane-Weiler, o qual leva em consideração o forte acoplamento entre a banda de condução e de valência e a mistura dos estados eletrônicos e de spin. Uma discussão detalhada das simetrias associadas com os níveis eletrônicos e as regras de seleção para transições ópticas intrabanda são obtidas, considerando as polarizações da luz incidente circular e linear. Calculam-se também as forças do oscilador óptico e seu correspondente espectro de absorção óptico para qualquer polarização.

PALAVRAS-CHAVE: Ponto quântico. Magneto-absorção intrabanda. Hamiltoniano k.p. Simetrias.
\end{abstract}

ABSTRACT: In this work discuss different aspects of the optical properties in a single CdTe spherical quantum dot in presence of a external magnetic field $B$ applied in the z-direction after perform a systematic study of the energy eigenvalues, wavefunctions and their dominant symmetries within the full $8 \times 8$ k.p Kane-Weiler theory that allows the inclusion of the conduction-valence band coupling and mixing of the electronic and spin states. A detailed discussion of the symmetries associated with the electronic levels and the selection rules for intraband optical transitions are derived by considering both circular and linear polarization of the incident light. We also calculated the optical oscillator strengths and their corresponding linear optical absorption spectra for any polarization..

KEYWORDS:

\section{INTRODUÇÃO}

Os estados da banda de condução de pontos quânticos semicondutores têm sido estudados experimentalmente através de tunelamento ressonante, espalhamento Raman e absorção intrabanda com luz infravermelha (SHUM, 1992). Este tipo de estudo é complementar às medidas baseadas em fotoluminescência e permite investigar separadamente os processos de relaxação dos elétrons e dos buracos (KLIMOV, 1999). Um estudo detalhado da absorção intra- e interbanda, nas diversas configurações da polarização da luz, pode permitir determinar, separadamente, o fator- $g$ do elétron e do buraco. Processos de relaxação do portador e uma identificação precisa das simetrias dos níveis Zeeman podem ser determinados. O objetivo deste trabalho é estudar, dentro da aproximação k.p de Kane-Weiler, a absorção intrabanda em um ponto quântico esférico (PQE) na presença de um campo magnético externo, para as diversas polarizações da luz incidente. Este estudo demonstra que o controle preciso e a identificação das transições ópticas intrabanda podem ser úteis para projetar e 
caracterizar estes novos sistemas. A escassez de resultados experimentais nesta área está relacionada à dificuldade em se isolar um único ponto quântico. Entretanto, Empedocles, Norris e Bawendi, (1997) e Sercel e Vahala (1990) foram capazes de realizar medidas de espectroscopia de fotoluminescência e de efeito Stark de confinamento quântico em um único nanocristal de CdSe. Esta nova técnica experimental pode permitir realizar estudos experimentais em magneto-óptica em PQE's.

\section{TEORIA}

É bem aceito que o espectro eletrônico em um campo potencial cristalino de uma dada nanoestrutura semicondutora pode ser bem descrito usando a teoria de multibandas k.p.

A identificação dos estados baseada nesta teoria fornece um forte fundamento para quase toda descrição detalhada da estrutura eletrônica em pontos quânticos (PRADO, 2003; SERCEL, 1990). Este método foi usado para estudar uma grande classe de propriedades ópticas, tais como absorção (PRADO, 2003; PAULA, 1999) ou espalhamento ressonante Raman (LÓPEZ-RICHARD, 1998), produzindo um bom acordo com os resultados experimentais.

Várias versões desta teoria foram formuladas e neste trabalho usa-se o modelo k.p $8 \times 8$, Kane-Weiler (KANE, 1957), o qual leva em consideração as interações entre as bandas $\Gamma_{6}, \Gamma_{8}, \Gamma_{7}$ exatamente e considera as contribuições das bandas remotas. A simetria inerente no Hamiltoniano k.p permite a separação do espaço de Hilbert em dois subespaços ortogonais. As funções de onda são expandidas em espinores de oito-componentes em cada subespaço de Hilbert, usando as soluções exatas dos termos da diagonal para cada tipo de portador. Cada componente do espinor tem a forma $f^{M}{ }_{n, L}(r, \Omega)=A_{n, L} j_{L}\left(\alpha^{L}{ }_{n} r / R\right) Y_{L}^{M}(\Omega)$. As funções de ondas $\psi_{I(I I)}$ para cada subespaço I e II satisfazem completamente a condição de contorno, $\psi_{\mathrm{I}(\mathrm{II})}(R)=0 \mathrm{e}$ são escritas como

$$
\left|\psi_{\text {I(II) }}^{M}\right\rangle=\sum_{L \geq M \mid}^{\infty}\left(\begin{array}{l|l}
f_{n, 2 L(2 L+1)}^{M}|e \uparrow\rangle \\
f_{n, 2 L+1(2 L)}^{M-1}|h h \uparrow\rangle \\
f_{n, 2 L+1(2 L)}^{M}|l h \uparrow\rangle \\
f_{n, 2 L+1(2 L)}^{M}|s O \uparrow\rangle \\
f_{n, 2 L(2 L+1)}^{M+1}|e \downarrow\rangle \\
f_{n, 2 L+1(2 L)}^{M+2}|h h \downarrow\rangle \\
f_{n, 2 L+1(2 L)}^{M+1}|l h \downarrow\rangle \\
f_{n, 2 L+1(2 L)}^{M+1}|s O \downarrow\rangle
\end{array}\right)
$$

O esquema de diagonalização da matriz foi desenvolvido ordenando o conjunto de funções envelope $f$ para valores crescentes da energia $E_{i}$, $i=1 \ldots N$. Portanto, a soma $\sum_{l}$ na expressão (1) pode ser trocada por $\sum_{E_{i}}$, com os números quânticos $L$ e $M$ fixos. Este procedimento permite selecionar somente as contribuições mais importantes para a formação de um dado estado. Obtém assim um ganho significativo na eficiência computacional, desde que as matrizes a serem diagonalizadas são muito menores do que as normalmente usadas em procedimentos padrão. Outros detalhes do esquema de diagonalização podem ser encontrados em (PRADO, 2003).

Para incluir os efeitos de um campo magnético externo $\mathbf{B}=(0,0, B)$, aplicado na direção- $\mathbf{z}$ deve-se modificar o Hamiltoniano por meio da substituição do operador $\mathbf{p}$ por $-i \hbar \nabla_{x, y, z} \pm(e / c) \mathrm{A}$, onde o sinal superior (inferior) é para elétrons (buracos). O gauge simétrico foi escolhido para o vetor potencial, A, para os elétrons e buracos $\mathrm{A}=\frac{1}{2} B r \sin (\theta)\left(-\sin \phi \hat{e}_{x}+\cos \phi \hat{e}_{y}\right)$. Quando um 
campo magnético externo é aplicado, dois efeitos podem ser identificados dependendo da intensidade do campo. Se o campo magnético é fraco, o efeito dominante é a separação dos estados de spin. O alcance do potencial de confinamento magnético é medido pelo raio ciclotrônico $I_{B}$, a separação do spin é dominante quando $I_{B}>R$. Por outro lado, se o campo magnético é forte, tal que $I_{B}<R$, importantes deformações da função de onda irão aparecer e serão formados os níveis de Landau. Este trabalho restringese ao caso $I_{B}>R$, onde a função de onda é fracamente afetada pelo campo magnético, permitindo o uso de um conjunto de funções parabólicas em $B=0$ como uma base apropriada para diagonalizar o Hamiltoniano.

\section{ABSORÇÃo ÓPTICA INTRABANDA}

O espectro de absorção óptico intrabanda foi calculado considerando um parâmetro de espalhamento homogêneo $\Gamma=20 \mathrm{meV}$, que pode ser associado com a presença de impurezas, estados de superfície, etc. A probabilidade das transições ópticas permitidas pela regra de seleção de dipolo foram calculadas em detalhes, possibilitando assim discutir o espectro de absorção óptico.

$\mathrm{Na}$ aproximação de dipolo elétrico, a força do oscilador é uma combinação dos elementos de matriz das transições ópticas

$$
\begin{aligned}
\left\langle\psi_{j, f}|\hat{\mathbf{e}} . \hat{\mathbf{P}}| \psi_{j^{\prime}, i}\right\rangle= & \left\langle f_{j, f} \mid f_{j^{\prime}, f}\right\rangle\left\langle\mu_{j}|\hat{\mathbf{e}} \hat{\mathbf{P}}| \mu_{j^{\prime}}\right\rangle+ \\
& \left\langle\mu_{j} \mid \mu_{j^{\prime}}\right\rangle\left\langle f_{j, f}|\hat{\mathbf{e}} \hat{\mathbf{P}}| f_{j^{\prime}, f}\right\rangle_{(2)}
\end{aligned}
$$

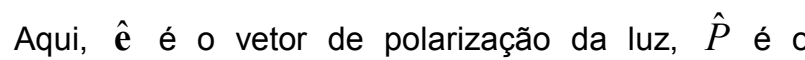
operador momentum, $f$ e $u$ são a função envelope e a função periódica de Bloch no ponto $\Gamma$ para cada portador, respectivamente. O primeiro termo do lado direito da expressão (2) permite obter as regras de seleção para as transições interbanda, e o segundo termo está relacionado com as transições interbanda.
Assim, para o caso intrabanda a expressão $\left\langle\mu_{j} \mid \mu_{j^{\prime}}\right\rangle\left\langle f_{j, f}|\hat{\mathbf{e}} \hat{\mathbf{P}}| f_{j^{\prime}, f}\right\rangle$ permite transições entre estados de diferentes paridades, ou equivalentemente, quando o estado inicial e final pertence a diferentes subespaços de Hilbert.

$$
\text { O termo }\left\langle\mu_{j} \mid \mu_{j}\right\rangle \text { é igual à função delta } \delta_{\mathrm{j}, \mathrm{j}},
$$
então transições entre estados com o mesmo número de spin são permitidos, a integral $\left\langle f_{j, f}|\hat{\mathbf{e}} . \hat{\mathbf{P}}| f_{j^{\prime}, f}\right\rangle$ fornece as regras de seleção para as polarizações circular e linear. Portanto, para as polarizações circulares $\hat{\mathbf{e}}^{ \pm}$, as regras de seleção são $\Delta M= \pm 1$ e $\Delta L$ $= \pm 1$. Para a luz polarizada linearmente $\hat{\mathbf{e}}^{z}$ tem-se: $\Delta M=0$ e $\Delta L= \pm 1$. Estas regras de seleção são válidas para transições ópticas intrabanda com ou sem campo magnético externo.

A absorção óptica intrabanda para a luz com polarização circular $\hat{\mathbf{e}}^{-}$é dada por

$$
\begin{aligned}
& \alpha(\hat{e}, \omega)=\frac{\alpha_{0} \Gamma}{\pi} \sum_{N_{e}, N_{e}, M} \frac{\left|F_{\mathrm{N}_{e}, M}^{N_{e}, M \pm 1}(\mathrm{I}, \mathrm{II})\right|^{2}}{\left[\left(E_{N_{e}, M}(\mathrm{I})-E_{N_{e}, M}(\mathrm{II})-\hbar \omega\right)^{2}-\Gamma^{2}\right]} \\
& +\frac{\left|F_{\mathrm{N}_{\mathrm{e}}, M}^{N_{e}, M+1}(\mathrm{II}, \mathrm{I})\right|^{2}}{\left[\left(E_{N_{e}, M}(\mathrm{II})-E_{N_{e}, M}(\mathrm{I})-\hbar \omega\right)^{2}-\Gamma^{2}\right]}
\end{aligned}
$$

e uma expressão equivalente pode ser escrita para a luz com polarização linear $\hat{\mathbf{e}}^{z}$

$$
\begin{aligned}
& \alpha\left(\hat{e}^{z}, \omega\right)=\frac{\alpha_{0} \Gamma}{\pi} \sum_{N_{c}, N_{e}, M} \frac{\left|F_{\mathrm{N}_{e}, M}^{N_{e}, M}(\mathrm{I}, \mathrm{II})\right|^{2}}{\left[\left(E_{N_{e}, M}(\mathrm{I})-E_{N_{e}, M}(\mathrm{II})-\hbar \omega\right)^{2}-\Gamma^{2}\right]} \\
& +\frac{\left|F_{\mathrm{N}_{e}, M}^{N_{e}, M}(\mathrm{II}, \mathrm{I})\right|^{2}}{\left[\left(E_{N_{e}, M}(\mathrm{II})-E_{N_{e}, M}(\mathrm{I})-\hbar \omega\right)^{2}-\Gamma^{2}\right]}
\end{aligned}
$$

Nas expressões acima, $\alpha_{0}$ é a constante de normalização, $\omega$ é a freqüência da luz incidente e $F_{\mathrm{N}_{\mathrm{e}}, M}^{N_{e}, M \pm 1}(\mathrm{I}, \mathrm{II})\left(F_{\mathrm{N}_{\mathrm{e}}, M}^{N_{e}, M}(\mathrm{I}, \mathrm{II})\right)$ é à força do oscilador óptico para as transições permitidas. 


\section{A. ABSORÇÃO ÓPTICA INTRABANDA COM $B=0$}

A FIG. 1, mostra a força do oscilador como função do raio $R$, para um $\mathrm{PQE}$ de CdTe, cujos parâmetros usados no cálculo são; a energia do gap $E_{g}=1,6069$ $\mathrm{eV}$, energia da interação spin-órbita $\Delta_{0}=0,953 \mathrm{eV}$, os parâmetros de Luttinger $\gamma_{1}^{L}=5,37, \gamma_{2}^{L}=1,67$, $\gamma_{3}^{L}=1,98$ (em unidades de $\frac{\hbar^{2}}{m_{0}}$ ), a não-parabolicidade da banda de condução $(1+2 F)=1,24$, a massa efetiva do elétron $m_{e}=0,091 m_{0}$, e o parâmetro de Kane de acoplamento das bandas de conduçãovalência $E_{p}=1,79 \mathrm{eV}$. A unidade de comprimento usada na figura é $1 \bar{A}=10^{-6} \mathrm{~cm}$.

Para o modelo de Hamiltoniano esférico, todas as três polarizações devem dar o mesmo resultado, já que todos os três eixos são idênticos. Certamente as propriedades ópticas para as polarizações, $\hat{\mathbf{e}}^{ \pm}$ficam idênticas para o modelo de Hamiltoniano, entretanto devem aparecer pequenas diferenças no espectro de absorção para $\hat{\mathbf{e}}^{z}$, como pode ser observado nas figuras a seguir.

As transições envolvidas foram obtidas da regra de seleção interbanda geral $\Delta \mathrm{M}=0, \pm 1$, como identificadas na Tabela 1.

Duas características qualitativas podem ser observadas nestas figuras, i) a força do oscilador apresenta uma suave variação com o raio do ponto e para algumas transições, e para raios bem definidos, a força do oscilador exibe uma brusca variação, ii) uma clara troca das forças ópticas entre as transições 1 e 2 podem ser observadas em aproximadamente $R=42 \AA$. Para pontos de tamanhos $R<42 \AA \hat{\text { a }}$ transição 1 é dominante, enquanto que a transição 2 é extremamente fraca. O mesmo comportamento é observado para as transições 1 e 2 e 3 e 4 para a polarização $\hat{\mathbf{e}}^{z}$. Estes resultados podem ser explicados, considerando que o estado final das transições mencionadas são os estados eletrônicos
$0(-1) e^{1}(I I)$ e $0(-1) e^{2}(I I)$ (o número entre parênteses é usado para representar estados degenerados). Estes níveis apresentam um anti-cruzamento próximo a $R=$ $42 \hat{\text {. }}$

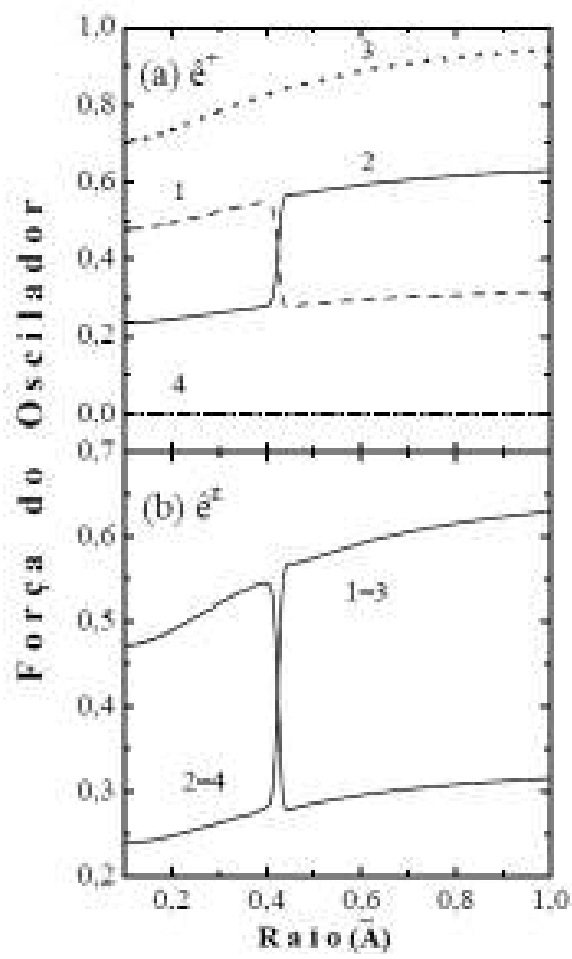

FIGURA 1. Força do oscilador como função do raio do ponto, considerando as polarizações $\hat{\mathbf{e}}^{+} \mathbf{e} \hat{\mathbf{e}}^{z}$. As transições mostradas estão identificadas na Tabela 1.

TABELA 1. Transições ópticas permitidas para luz incidente com polarizações $\hat{\mathbf{e}}^{-}, \hat{\mathbf{e}}^{+} \mathrm{e} \hat{\mathbf{e}}^{z}$. Os números na primeira coluna estão relacionados com as figuras. 1 ,

$$
2,3 \text { e } 4 \text {. }
$$

\begin{tabular}{|c|c|c|}
\hline & $\hat{\mathbf{e}}^{-}$ & $\hat{\mathbf{e}}^{z}$ \\
\hline 1 & ${ }_{-1} e^{1}(I I) \leftarrow{ }_{0} e^{1}(I)$ & ${ }_{1} e^{1}(I I) \leftarrow{ }_{0} e^{1}(I) \quad{ }_{0} e^{1}(I I) \leftarrow{ }_{0} e^{1}(I)$ \\
\hline 2 & ${ }_{-1} e^{2}(I I) \leftarrow{ }_{0} e^{1}(I)$ & ${ }_{0} e^{1}(I I) \leftarrow{ }_{-1} e^{1}(I) \quad{ }_{0} e^{2}(I I) \leftarrow{ }_{0} e^{1}(I)$ \\
\hline 3 & ${ }_{-2} e^{1}(I I) \leftarrow{ }_{-1} e^{1}(I)$ & ${ }_{0} e^{2}(I I) \leftarrow{ }_{-1} e^{1}(I){ }_{-1} e^{1}(I I) \leftarrow{ }_{-1} e^{1}(I)$ \\
\hline 4 & ${ }_{-2} e^{2}(I I) \leftarrow{ }_{-1} e^{1}(I)$ & ${ }_{1} e^{2}(I I) \leftarrow{ }_{0} e^{1}(I) \quad{ }_{-1} e^{2}(I I) \leftarrow{ }_{-1} e^{1}(I)$ \\
\hline
\end{tabular}




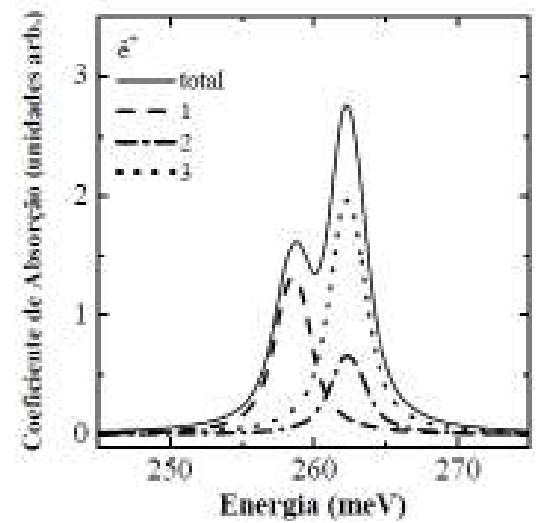

FIGURA 2. Espectro de absorção para um PQE de CdTe com $R=30 \AA$, para a polarização da luz incidente $\hat{\mathbf{e}}^{+}$. As linhas 1, 2 e 3 representam as três contribuições para o espectro óptico total (linha cheia).

Neste tamanho de ponto os estados trocam seu caráter e, de acordo com as regras de seleção, a força do oscilador devem refletir esta mudança e promove algumas transições. A FIG. 2 mostra o espectro de absorção calculado para a polarização $\hat{\mathbf{e}}^{+}$para

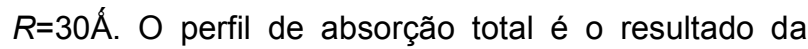
adição de todas as três possíveis contribuições, listadas na tabela 1. A forma do perfil de absorção para $\hat{\mathbf{e}}^{-}$é exatamente o mesmo que para $\hat{\mathbf{e}}^{+}$e $\hat{\mathbf{e}}^{z}$. Este fato é uma conseqüência da simetria espacial do problema, o qual garante que os níveis de energia são degenerados com respeito ao número quântico $M$. Uma rápida inspeção da Eq. (1) permite mostrar que os espectros para $\hat{\mathbf{e}}^{+} \mathrm{e} \hat{\mathbf{e}}^{-}$são quantitativamente semelhantes. Este comportamento deve ser esperado, desde que as funções base, usadas na determinação das transições permitidas para as polarizações circulares, resultam em um espectro de energia degenerado. Qualquer modificação na simetria esférica, induzida por campos externos tais como, o elétrico ou o magnético ou do desvio da forma esférica podem quebrar a degenerescência- $(2 L+1)$ e criar diferenças significativas entre os espectros de absorção $\hat{\mathbf{e}}^{+}, \hat{\mathbf{e}}^{-}$e $\hat{\mathbf{e}}^{z}$, como pode ser visto na seção seguinte, onde foi aplicado um campo magnético externo $B$ no sistema.

\section{B. ABSORÇÃO ÓPTICA INTRABANDA COM $B \neq 0$}

Como mencionado acima, a presença de um campo magnético, não modifica as regras de seleção intrabanda para as polarizações $\hat{\mathbf{e}}^{ \pm}$. Nas figuras $3(a)$ e 3(b) tem-se as forças do oscilador calculadas com função do campo magnético para um $P Q E$ de raio $R=30 \AA$ para as polarizações circulares. Em aproximadamente 1,6 $\mathrm{T}$ para $\hat{\mathbf{e}}^{+}$, pode-se observar um cruzamento na força do oscilador associado com as transições 1 e 2 . Este cruzamento não é abrupto como aquele da FIG. 1, a força do oscilador varia suavemente com o campo magnético.

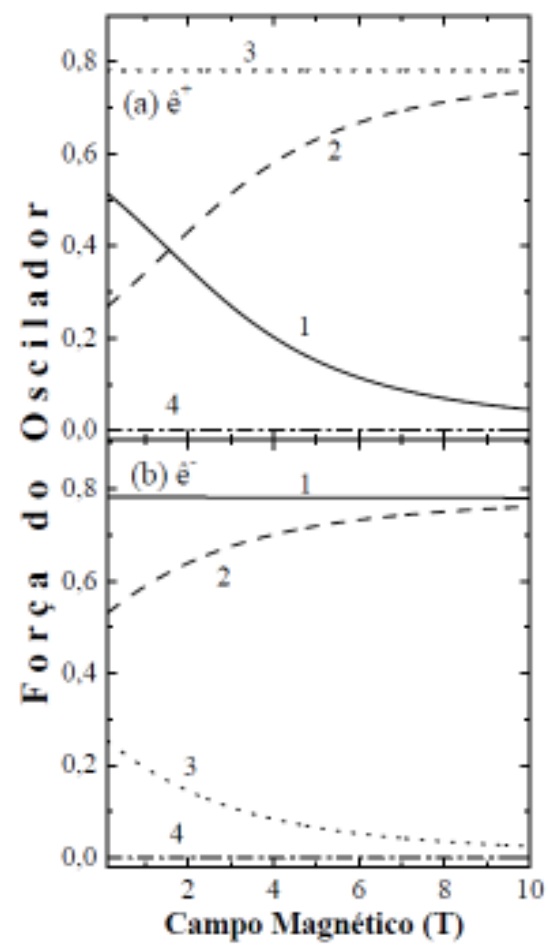

FIGURA 3. Força do oscilador como função do campo magnético, considerando as polarizações circulares a direita (a) $\hat{\mathbf{e}}^{+}$e a esquerda (b) $\hat{\mathbf{e}}^{-}$.

As transições mostradas estão listadas na Tabela 1.

Portanto, além da separação dos picos de absorção devido à quebra da degenerescência em $M$, o 
espectro de absorção total para $\hat{\mathbf{e}}^{+}$mostra uma continua troca entre as forças do oscilador para as transições 1 e 2 . Nas figuras 4(a) e 4(b) tem-se os espectro de magneto absorção para $\hat{\mathbf{e}}^{+} \mathrm{e} \hat{\mathbf{e}}^{-}$, calculados para um campo magnético fixo de 3,5T.

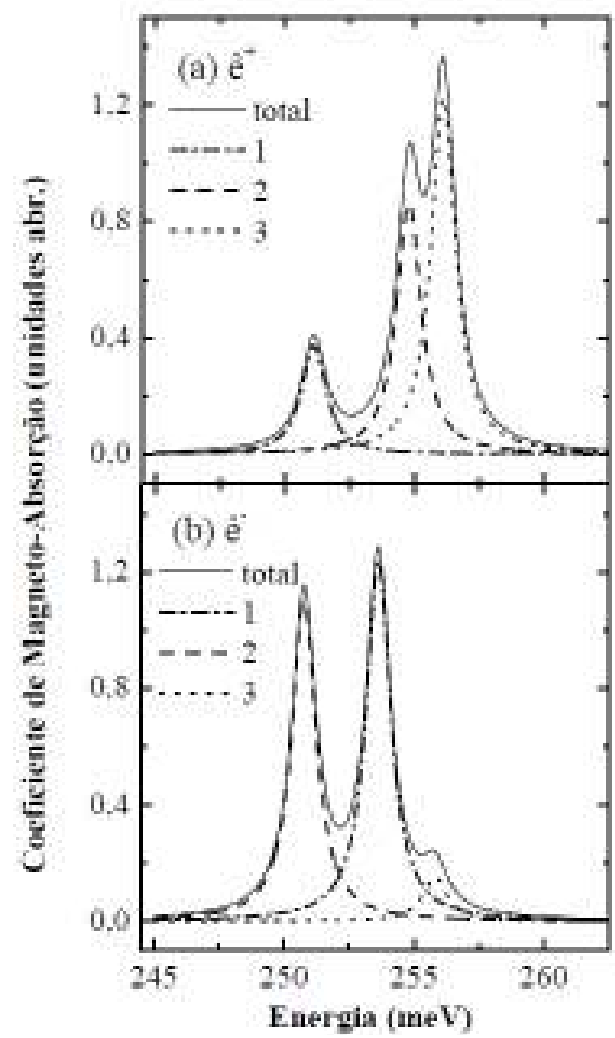

FIGURA. 4. Espectro de absorção para um PQE de CdTe com $R=30 \AA ̊$ com campo magnético. As linhas 1 , 2 e 3 representam somente as três contribuições para o espectro óptico total (linha cheia). Observe-se o texto para mais detalhes.

É necessário enfatizar alguns fatos interessantes nestas figuras: i) a quebra da degenerescência em $M$ é claramente visível nas transições consideradas, ii) os picos de magneto-absorção, que compõem o pico total, movem-se em direção a posições de menor energia refletindo a variação da energia de transição com o campo magnético. Considerando que as regras de seleção somente permitem transições com $\Delta M= \pm 1$, o comportamento da energia de transição depende essencialmente dos efeitos de acoplamento com a banda de valência e com as variações sobre o fator- $g$ eletrônico efetivo. De acordo com as posições dos picos nas figuras $4(a)$ e $4(\mathrm{~b})$ e fazendo a diferença entre $\hat{\mathbf{e}}^{+} \mathrm{e} \hat{\mathbf{e}}^{-}$, absorbância (que se assemelha a técnica de dicroísmo magnético circular), é possível notar que o valor do fator-g eletrônico efetivo para baixas energias é negativo. Esta capacidade de modular o fator de Landè permite controlar uma grande variedade de propriedades magneto-ópticas.

\section{Conclusões}

Usando da aproximação de massa efetiva multibandas k.p estudou-se as principais características da estrutura eletrônica e das transições ópticas intrabanda em um único PQE na presença de um campo magnético externo. Mostraram-se as forças do oscilador e os cálculos de absorção, onde os efeitos de não-homogeneidade da distribuição de tamanhos foram negligenciados e a interação radiação-matéria foi tratada na aproximação dipolar. Os resultados são um ponto de partida para uma discussão completa e rigorosa de outras propriedades magneto-ópticas em pontos quânticos semicondutores e a possibilidade de observar excitações com diferentes polarizações.

\section{REFERÊNCIAS}

EMPEDOCLES, S. A., NORRIS, D. J. e BAWENDI, M. G., Photoluminescence spectroscopy of single CdSe nanocrystallite quantum dots, Physical Review Letters, Vol. 77, 3873 (1996).
KANE, E. O., Semiconductor and Semimetals, Willardson, R.K. e Beer, A.C., eds. (Academic Press, New York, 1966), vol. 1, p.75.

KLIMOV, V. I., Schwarz, Ch. J., McBRANCH, C. J. S. D., LEATHERDALE, C. A. e BAWENDI, M. G., 
Ultrafast dynamics of inter- and intrabanda transitions in semiconductor nanocrystals: Implications for quantum dot lasers, Physical Review, Vol. 60, R2177, 1999.

LÓPEZ-RICHARD, V., MARQUES, G. E., DRAKE, J. e TRALLERO-GINER, C., Resonant Raman scattering in a magnetic Field assisted by Fröhlich interaction in a zinc-blende-type semiconductors, Physical Review B, Vol. 58, 16136, 1998.

PAULA, de A. M., OLIVEIRA, C. R. M., MARQUES, G. E., COHEN, A. M., ISLAM, M. N. e CESAR, C. L., Interband and intersubband absorption in $\mathrm{HgCdTe}$ multiple quantum Wells, Physical Review B, Vol. 59, 10158, 1999.

PRADO, S. J.; TRALLERO-GINER, C.; ALCALDE, A. M.; LÓPEZ-RICHARD, V. e MARQUES, G. E., Magneto-Optical properties of nanocrystal: Zeeman splitting, Physical Review B, Vol. 67, p. 165306, 2003.
SERCEL, P. C. e VAHALA, K. J., Analytical formalism for determining quantum-wire and quantum-dot band structure in the multiband envelope-function approximation, Physical Review. B, Vol. 42, 3690, 1990.

SHUM, K., WANG, W. B. e ALFANO, R. R. Observation of the $1 P$ excitonic states in $\mathrm{Cd}(\mathrm{S}, \mathrm{Se})$ glass quantum dots, Physical Review Letters. Vol. 68, 3904, 1992. 